\title{
Análisis de workflows científicos mediante el paradigma de las U-RDF-PN
}

\author{
E. González López de Murillas, J. Fabra \\ Grupo de Integración de Sistemas Distribuidos y Heterogéneos (GIDHE) \\ Instituto de Investigación en Ingeniería de Aragón (I3A) \\ Universidad de Zaragoza, Mariano Esquillor s/n, 50018, Zaragoza, Spain. \\ Tel. +34-976761000 ext. 5458, Fax +34-976762043, e-mail: edugonza@unizar.es
}

\begin{abstract}
La computación científica ha ganado un creciente interés en los últimos años en áreas afines a las ciencias de la vida. Los workflows científicos son un tipo especial de workflow utilizados en escenarios de grandes dimensiones y gran complejidad computacional, como modelos climáticos, estructuras biológicas, química, cirugía o simulación de desastres, por ejemplo. La computación científica ha mejorado progresivamente a través de la introducción de nuevos paradigmas y tecnologías para poder abordar desafíos cada vez más complejos. Esta línea de investigación se centra en la adición de aspectos semánticos al área de la computación científica, aportando un método de model checking basado en la introducción de aspectos y anotaciones semánticas tanto en los modelos como en las fórmulas que deben verificarse. La adición de aspectos semánticos flexibiliza la especificación y facilita la integración de servicios remotos y heterogéneos. Como punto de partida, el desarrollo del toolkit COMBAS (COmprobador de Modelos Basado en Semántica) proporciona un entorno que integra herramientas para la verificación de modelos que incluyen información semántica y la navegación por las estructuras resultante del proceso. Para la descripción de modelos de workflows científicos se utiliza una clase de Redes de Petri de alto nivel anotadas con información semántica, las U-RDF-PN, en las que el Grupo de Integración de Sistemas Distribuidos y Heterogéneos (GIDHE), donde el autor desarrolla su línea de investigación, tiene una amplia y demostrada experiencia. Finalmente, el enfoque propuesto se aplicará a una serie de problemas reales de relevancia en el mundo científico para demostrar su utilidad y viabilidad.
\end{abstract}

